\section{Role of viruses in febrile convulsions}

Sir,

Lewis et al., ${ }^{1}$ reporting on viruses isolated from children with febrile convulsions, found adenovirus in the CSF in 3 cases and parainfluenza virus in the CSF of another.

We have studied the viruses in the CSF of children aged under 24 months with febrile convulsions, making use of a virology service in the main hospital, with a technician able to take cultures and inoculate them into the cell lines at the bedside.

Viruses were isolated from the CSF in 9 cases out of 66 (herpes simplex type 1 in 1 case, type 2 in 4 cases, type uncertain in 2 cases; echovirus (not typed) 1 case; enterovirus (not typed) 1 case). None of the 9 cases showed clinical laboratory evidence of meningeal irritation. Neurodevelopmental follow-up after 2 years was normal in each case. All were given sodium valproate to prevent recurrences. ${ }^{2}$

No viruses were isolated from 25 random CSF specimens of patients of the same age without nervous or infectious disease.

The significance of these preliminary findings is being examined.

\section{References}

1 Lewis H M, Parry J V, Parry R P, et al. Role of viruses in febrile convulsions. Arch Dis Child 1979; 54: 869-76.

2 Cavazzuti G B. Prevention of febrile convulsions with dipropylacetate. Epilepsia $1975 ; 16$ : 647-8.

F SoUADRINI AND F TAPARELLI Laboratory for Virus Research, Department of Infectious Diseases,

A Nalin and G B Cavazzuti Department of Paediatrics and Neonatal Medicine, University of Modena Medical School, Modena, Italy

\section{Dr Lewis and co-workers comment:}

These observations support our hypothesis that a febrile convulsion depends on the effect of a viraemia on the central nervous system. Herpes simplex was identified in 7 out of 9 cases, but in our study we did not isolate this virus from systemic sites (CSF, blood, or urine) in any child. We wonder whether Dr Squadrini's patients had clinical evidence of herpetic infection or if virus was isolated from sites other than the CSF. We await further details with interest.

Widespread early herpes simplex infection is reported to be common in children living in underdeveloped communities in Africa. We would expect to find a different spectrum of viruses in Sicily compared with Harrow, England, and a febrile convulsion could be a response to systemic invasion by many different viruses.

Helen Lewis, D A J Tyrrell, AND H B VALMAN Northwick Park Hospital and Clinical Research Centre, Harrow, Middx

\section{Tomography of neonates with abnormal ears}

Sir,

Atresia of the external auditory meatus is not rare and should always be diagnosed at birth, but tomographic examinations of the petrous temporal bones are rarely performed in the neonatal period. We should like to make a case for early radiological assessment of children with certain ear deformities.

Operations to improve the hearing through creation of a new meatus and reconstruction of the hearing mechanism of the middle ear are not now generally performed on unilateral cases. However, children with bilateral atresia should be operated on at age 2 years. ${ }^{1}$ The feasibility of surgery depends on the presence of a normal inner ear and a reasonable middle ear cavity. This must be assessed by expert interpretation of high quality multi-directional tomograms, which can often be obtained in the first few months of life with no more sedation than a large feed, and with an acceptable radiation dose to the eyes.

Malformations of the pinna are often associated with lesions of the middle and inner ear, and a birth incidence as high as $1 \%$ has been estimated for malformations of the external ear, including branchial sinuses. ${ }^{2}$ Several syndromes are now known to be associated with abnormalities of the aural conduction mechanism. Children with the Treacher Collins syndrome, for instance, often have ossicular abnormalities with normal eardrums. ${ }^{3}$ We are currently trying to determine which rare syndromes are associated with a high incidence of conduction abnormalities; this aspect has been poorly documented.

Objective assessment of deaf children is improving rapidly with evoked response audiometry, but the best method, electrocochleography, is not possible if there is meatal atresia. Obviously tomography cannot assess cochlear function or show deficiencies in the hearing end organ, but it will give an accurate demonstration of the bony cochlea. It will show not only major deformities incompatible with hearing, but also minor anomalies of the central bony spiral where some cochlear function may be present.

The 'at risk register' ensures that most deaf children now have continuing assessment and education from age one year, but we believe that in selected cases tomography of the petrous temporal bones has an important part to play. Ideally the examination should be in the neonatal period, with the films, report, and conclusions available for continuing assessment.

\section{References}

1 Livingstone G. Congenital ear abnormalities due to thalidomide. Proc $R$ Soc Med 1965; 58: 493-7.

2 Melnick M, Myrianthopoulos N C. External ear malformations: epidemiology, genetics, and natural history. Birth Defects 1979; 15: No 9, 1-140.

3 Phelps P D, Poswillo D, Lloyd G A S. The ear deformities in mandibulofacial dysostosis. Clin Otolaryngol 1980. In press.

Peter D Phelps and Glyn A S Lloyd Royal National Throat, Nose, and Ear Hospital, Gray's Inn Road, London WCIX $8 D A$ 